\title{
The appropriateness of invasive ventilation in COVID-19 positive cancer patients: the hardest decision for oncologists.
}

Michele Ghidini $^{1}$; Alice Indini ${ }^{1}$; Erika Rijavec ${ }^{1}$; Claudia Bareggi ${ }^{1}$; Monica Cattaneo ${ }^{1,2}$; Barbara Galassi ${ }^{1}$; Donatella Gambini ${ }^{1}$; Francesco Grossi ${ }^{1}$.

\section{Corresponding author:}

Dr. Francesco Grossi,

Medical Oncology Unit, Fondazione IRCCS Ca’ Granda Ospedale Maggiore Policlinico,

Via Francesco Sforza 35, 20122, Milano, Italy

Phone: +39-0255032660; fax: +39-0255032659

E-mail: francesco.grossi@policlinico.mi.it

\begin{abstract}
Over the last two months, as oncology specialists, we have frequently been contacted for estimating prognosis for cancer patients affected by COVID-19 infection. Until now, there have been no clear markers to guide decision making regarding the appropriateness of invasive ventilation in cancer patients affected by COVID-19 infection.

Therefore, we developed a practical tool encompassing a prognostic score. We aimed at identifying a subgroup of patients likely to have a better outcome and therefore may be potential candidates for invasive ventilation, "The Milano Policlinico ONCOVID-ICU score". The score is composed by three groups of variables: patient's characteristics such as sex, age, BMI and comorbidities; oncological variables (treatment intent, life expectancy, on or offtreatment status) and clinical parameters in association with laboratory values (SOFA score and D-dimer). The SOFA score includes six different clinical parameters and during the first few days of ICU admissions has an important prognostic role.
\end{abstract}


The oncological history should never represent, per se, a contraindication to intensive care and must be considered together with other variables, such as laboratory values, clinical parameters and patient characteristics, in order to make the hardest but best possible choice. The Milano Policlinico ONCOVID-ICU score, to our knowledge, is the first prognostic score proposed in this setting of patients and may be a useful tool to assess the prognosis of cancer patients being in this critical condition.

\section{Keywords}

COVID-19; invasive ventilation; cancer; ICU; ONCOVID-ICU; Milano Policlinico; SOFA score; ARDS.

Over the last two months, as oncology specialists, we have frequently been contacted to estimate prognosis and pro-actively make treatment escalation plans for cancer patients affected by COVID-19 infection. In the case of acutely deteriorating patients, such decisions often have to be made within a short space of time and can be challenging. Our Hospital Maggiore Policlinico of Milan is located in the centre of Milan and has a large emergency room, thus being able to accommodate patients from many different centres that don't have access to such services. Since the pandemic began, our hospital has been totally re-structured in order to cope with the increased case load. So far, patients with COVID-19 and its complications have occupied more than 500 beds (half of total beds capacity).

One Wednesday morning, we were contacted by a colleague who was caring for patients with COVID-19 in a different hospital in Milan. He wanted to discuss the ceiling of treatment for a mutual patient who was admitted with acute respiratory failure secondary to COVID-19 induced pneumonia and had increasing oxygen requirements. Is invasive mechanical ventilation appropriate in patients with active cancer? Our colleague instinctively proposed a 
palliative strategy without the use of C-PAP or invasive ventilation. Why? Our 65 year-old patient had a history of recurrent pancreatic cancer treated with single agent capecitabine for six months after eight cycles of second line chemotherapy with capecitabine and oxaliplatin and disease stability. Her progression-free interval from the beginning of second line therapy is approaching one year, overcoming our expectations and average median survival. She was last reviewed in our outpatient clinic on $13^{\text {th }}$ of March when she was well with an ECOG performance status (PS) of zero and no treatment related toxicities to report.

We only had a short moment to gather our thoughts and remind ourselves of the patient's clinical case. Some minutes later we were expected to make the ultimate decision for our patient: was further ventilatory support appropriate or not? Was our colleague right to suggest palliative care only? We took into consideration her age, good PS before COVID-19 infection, lack of significant co-morbidities, the unusually long progression-free interval suggesting favourable disease biology and the good tolerability of her recent chemotherapy. We recommended full escalation of treatment from C-PAP to invasive ventilation if deemed necessary. Were we wrong? We were not aware of any tools to support us with this tough decision.

It has been reported that the mortality rate of cancer patients admitted to European intensive care units (ICUs) is similar to that of those without cancer (20\% vs. $18 \%$, respectively). However, medical patients with advanced cancer have double the hospital mortality rate of surgical patients with cancer (41 vs 21\%, p<0.001).[1] Prospective observational data showed that even high-risk cancer patients may benefit from an early admission to ICUs, especially if treated before the onset of organ dysfunction. In these patients, a "full-code management" without limitations of ICU resources should be done for the first days (ideally $\geq 5$ days), because prognosis can't be estimated until this monitoring period has passed.[2,3] A recent systematic review attempted to establish a consensus on indications for intubation in cancer 
patients. Over the years, there has been an improvement in the outcome of cancer patients admitted to the ICU, with an average survival of $32.4 \%$ and a long-term survival of $10.2 \%$.[4] Among the risk factors for short-term mortality: age, severity and number of failing organs, presence of acute respiratory failure, PS, comorbidities and stage of the disease play a fundamental role.[3,4] Moreover, the Sequential Organ Failure Assessment (SOFA) score was reported as one of the major predictors of outcome for cancer patients admitted to ICU.[4] The SOFA score includes six different clinical parameters: the respiratory $\mathrm{PaO}_{2} / \mathrm{FIO}_{2}(\mathrm{P} / \mathrm{F})$ ratio, presence of ventilatory assistance, blood pressure, platelet count, the Glasgow Coma Score scale, bilirubin and creatinine levels (Table 1). Sequential evaluation of SOFA score during the first few days of ICU admissions has an important prognostic role. In the case of a stable or increase in the SOFA score in the first hours after admission to ICU, the reported death rates are $37 \%$ for a score between 2 and 7, 60\% with an initial score of $8-11$ and $91 \%$ in case of a score $>11$. On the contrary, for initial scores $<11$, a decreasing value is associated with a mortality rate of $6 \%$.[5]

Among predictors of poor outcome, the etiology of respiratory failure must be taken into consideration as well. While pulmonary edema in cancer patients has a reasonably good prognosis, infection is a predictor of poor outcome.[4] COVID-19 infection and subsequent pneumonitis can cause a condition of hypoxiemic respiratory failure and acute respiratory distress syndrome (ARDS), requiring oxygen and ventilation therapies, admittance to ICU in $15-20 \%$ of cases and, in approximately $3.2 \%$ of cases, intubation and invasive ventilation.[6] A prospective Chinese series of COVID-19 positive patients reported a statistically significant increase in terms of severe events requiring ICU admittance, invasive ventilation or death in cancer patients compared with cancer-free cases (39 vs $8 \%, \mathrm{p}=0.0003$ ). Moreover, patients undergoing surgery or chemotherapy for cancer in the previous months had a higher risk of clinically severe events when compared to patients who did not have these treatments (75 vs 
43\%).[7] Risk factors for mortality in adult inpatients with COVID-19 have been described in a Chinese retrospective multicentre cohort study. Median age of the population was 56 years. Multivariable regression identified older age (odds ratio [OR] 1.10, p=0.0043), higher SOFA score (OR 5.65, $\mathrm{p}<0.0001)$ and d-dimer greater than $1 \mu \mathrm{g} / \mathrm{mL}(\mathrm{OR} 18.42, \mathrm{p}=0.0033)$ on admission as significant prognostic factors for survival.[8]

Identification of prognostic factors for critically ill cancer patients following infection with COVID-19 is challenging but essential in order to define appropriate candidates for ICU admission and intubation. Many variables are present and most of the available series are mainly from the Chinese experience. Total number of cases, number of cases admitted to ICUs, median age of diagnosis, and mortality rates are far different from what was registered in Western European countries. Globally, median age of patients diagnosed with COVID-19 infection in Europe is higher than in China. Moreover, at the time of writing, Spain, Italy, UK and France have registered a mortality rate of between 10 and $13 \%$ compared to approximately 5\% registered in China, whilst the rate of ICU admission remains similar at 2$3 \%$ [9]. These differences may be justified at least partially by higher number of tests performed in China and the difference in median age of the population. Age is an important factor to take into consideration before admitting a cancer patient into the ICU. However, chronological age alone is a poor indicator of the physiological and functional status of a cancer patient and should not be considered as the main prognostic factor for treatment decisions in oncology.[10] Indeed, many patients over the age of 70 have an excellent PS and are suitable candidates for oncological treatment.

Until now, there have been no clear markers to guide decision making regarding the appropriateness of invasive ventilation in cancer patients affected by COVID-19 infection. Therefore, we developed a practical tool which encompasses a prognostic score in order to identify a subgroup of patients likely to have a better outcome and therefore may be potential 
candidates for invasive ventilation. The Milano Policlinico ONCOVID-ICU score includes three different groups of variables. In the first group we include sex, age, body mass index (BMI) and comorbidities. In a previous series, male sex was identified as an independent risk factor associated with worse prognosis and lack of clinical improvement in COVID-19 patients admitted to hospital (OR $=0.486, \mathrm{p}=0.001) \cdot[11]$ Moreover, we included BMI and comorbidities as they can be limiting factors for oncological treatment, and were reported as risk factors for short-term mortality in critically ill cancer patients requiring invasive ventilation. $[3,4]$ Old age was reported as one of the poor prognostic factors for survival in COVID-19 inpatients.[8] The second group includes oncological variables, such as the treatment intent (adjuvant or metastatic), life expectancy in months and availability of further treatment lines. Furthermore, we included the SOFA score and the d-dimer values, previously reported as risk factors for mortality in the presence of COVID-19 infection.[8] We identified three different groups (low, intermediate and high risk). We recommend that patients with a low risk score should be offered invasive procedures if necessary, while high-risk patients are best managed with best supportive care. Patients in the intermediate-risk group deserve a case-by-case discussion to derive a decision (Table 2). This division is arbitrary and the score needs further validation. We aim to validating our score by retrospectively assessing the clinical history of all cancer patients admitted to Milano Hospital Maggiore Policlinico's ICU. During the COVID-19 pandemic, cancer patients are facing not only higher risks of infections but also a lack of clear guidance from their treating physicians. Uncertainty regarding the safety of treatments (e.g. immunotherapy) in times of infection is a major topic of discussion. Furthermore, a considerable proportion of oncology patients may experience clinical deterioration due to the worsening course of the infection. These cases require a comprehensive evaluation before considering ICU admission and intubation. The oncological history should never represent, per se, a contraindication to intensive care and must be 
considered together with other variables, such as laboratory values, clinical parameters and patient characteristics, in order to make the hardest but best possible choice.

\section{References}

1 Taccone F, Artigas AA, Sprung CL, et al. Characteristics and outcomes of cancer patients in European ICUs. Crit Care 2009;13:R15. doi:10.1186/cc7713

2 Lecuyer L, Chevret S, Thiery G, et al. The ICU Trial: A new admission policy for cancer patients requiring mechanical ventilation*. Crit Care Med 2007;35:808-14. doi:10.1097/01.CCM.0000256846.27192.7A

3 Schellongowski P, Sperr WR, Wohlfarth $\mathrm{P}$, et al. Critically ill patients with cancer: chances and limitations of intensive care medicine-a narrative review. ESMO Open 2016;1:e000018. doi:10.1136/esmoopen-2015-000018

4 Huaringa AJ, Francis WH. Outcome of invasive mechanical ventilation in cancer patients: Intubate or not to intubate a patient with cancer. J Crit Care 2019;50:87-91. doi:10.1016/j.jcrc.2018.11.014

5 Ferreira FL. Serial Evaluation of the SOFA Score to Predict Outcome in Critically Ill Patients. JAMA 2001;286:1754. doi:10.1001/jama.286.14.1754

6 Meng L, Qiu H, Wan L, et al. Intubation and Ventilation amid the COVID-19 Outbreak. Anesthesiology 2020;:1. doi:10.1097/ALN.0000000000003296

7 Liang W, Guan W, Chen R, et al. Cancer patients in SARS-CoV-2 infection: a nationwide analysis in China. Lancet Oncol 2020;21:335-7. doi:10.1016/S1470-2045(20)30096-6

8 Zhou F, Yu T, Du R, et al. Clinical course and risk factors for mortality of adult inpatients with COVID-19 in Wuhan, China: a retrospective cohort study. Lancet 2020;395:105462. doi:10.1016/S0140-6736(20)30566-3

9 From COVID-19/Coronavirus, facts and figures. Website: https://www.statista.com/page/covid-19-coronavirus. Accessed: 19 $9^{\text {th }}$ April 2020.

10 Soto-Perez-de-Celis E, Li D, Yuan Y, et al. Functional versus chronological age: geriatric assessments to guide decision making in older patients with cancer. Lancet Oncol 2018;19:e305-16. doi:10.1016/S1470-2045(18)30348-6

11 Zhang J, Wang X, Jia X, et al. Risk factors for disease severity, unimprovement, and mortality of COVID-19 patients in Wuhan, China. Clin Microbiol Infect Published Online First: April 2020. doi:10.1016/j.cmi.2020.04.012 
Acknowledgements: Authors want to thank Dr. Shelize Khakoo for linguistic revision.

Authors' contributions: MG wrote the manuscript with imput from FG and AI. All authors read and approved the final manuscript.

Funding: Financed by Italian fiscal contribution "5x1000" 2016 devolved to Fondazione IRCCS Ca' Granda Ospedale Maggiore Policlinico.

Availability of data and materials: Not applicable.

Ethics approval and consent to partecipate: Not applicable.

Consent for publication: Not applicable.

Competing interests: The authors declare they have no conflict of interest.

Author details: ${ }^{1}$ Medical Oncology Unit, Fondazione IRCCS Ca' Granda Ospedale Maggiore Policlinico, Milano, Italy. ${ }^{2}$ Department of Medicine (DAME), University of Udine, Udine, Italy. 
Table 1. The SOFA score

\begin{tabular}{|c|c|c|c|c|c|}
\hline \multirow{2}{*}{ Variables } & \multicolumn{5}{|c|}{ SOFA Score } \\
\hline & $\mathbf{0}$ & 1 & 2 & 3 & 4 \\
\hline \begin{tabular}{l}
\multicolumn{1}{c}{ Respiratory } \\
$\mathrm{PaO}_{2} / \mathrm{FIO}_{2}(\mathrm{P} / \mathrm{F})$ \\
$\mathrm{mmHg}$
\end{tabular} & $>400$ & $\leq 400$ & $\leq 300$ & $\leq 200 *$ & $\leq 100 *$ \\
\hline $\begin{array}{l}\text { Coagulation } \\
\text { Platelets } \\
\text { x } 10^{9} / \mu \mathrm{g}\end{array}$ & $>150$ & $\leq 150$ & $\leq 100$ & $\leq 50$ & $\leq 20$ \\
\hline $\begin{array}{c}\text { Liver } \\
\text { Bilirubin } \mathrm{mg} / \mathrm{dL}\end{array}$ & $<1.2$ & $1.2-1.9$ & $2.0-5.9$ & $6.0-11.9$ & $>12$ \\
\hline $\begin{array}{c}\text { Cardiovascular } \\
\text { Hypotension }\end{array}$ & $\begin{array}{c}\text { No } \\
\text { hypotension }\end{array}$ & $\begin{array}{c}\text { Mean } \\
\text { arterial } \\
\text { pressure }< \\
70 \mathrm{mmHg}\end{array}$ & $\begin{array}{c}\text { Dopamine } \leq 5 \\
\text { or dobutamine } \\
\text { (any dose) }\end{array}$ & $\begin{array}{c}\text { Dopamine }>5 \text {, } \\
\text { epinephrine } \leq 0.1 \text {, or } \\
\text { norepinephrine } \leq \\
0.1\end{array}$ & $\begin{array}{c}\text { Dopamine }>15, \\
\text { epinephrine }>0.1 \text { or } \\
\text { norepinephrine }> \\
0.1\end{array}$ \\
\hline $\begin{array}{l}\text { Central nervous } \\
\quad \text { system } \\
\text { Glasgow Coma } \\
\text { Score Scale }\end{array}$ & 15 & $13-14$ & $10-12$ & $6-9$ & $<6$ \\
\hline $\begin{array}{l}\text { Renal } \\
\text { Creatinine } \\
\mathrm{mg} / \mathrm{dL}\end{array}$ & $<1.2$ & $1-2-1.9$ & $2.0-3.4$ & $3.5-4.9$ & $>5$ \\
\hline
\end{tabular}

* with ventilatory support 
Table 2. The Milano Policlinico ONCOVID-ICU score

\begin{tabular}{lc}
\hline \multicolumn{1}{c}{ Variables } & Score \\
\hline Patient characteristics & \\
Sex & $\mathrm{F}=0$ \\
$\mathrm{M}=1$ \\
Age & $<70=0$ \\
& $\geq 70=1$ \\
BMI & $<30=0$ \\
& $\geq 30=1$ \\
Comorbidities & NO $=0$ \\
& YES $=1$ \\
& YES $>1=2$ \\
\hline
\end{tabular}

\section{Oncological variables}

\begin{tabular}{l} 
Treatment intent \\
Life expectancy \\
Patient on treatment \\
\hline Clinical parameters + \\
laboratory values
\end{tabular}

Curative $=0$

Palliative $=1$

\section{Categories of risk for patients}

Score < 4: Low Risk

ICU admission and invasive ventilation.

\section{Score 4-6: Intermediate Risk \\ Case-by-case evaluation for} appropriateness of ICU admission and invasive ventilation.

\section{Score $\geq 7$ : High Risk}

Palliative treatment.

SOFA SCORE

$>6 \mathrm{mo}=0$

$<6 \mathrm{mo}=1$

$$
\mathrm{YES}=1
$$

$2-7=0$

$\geq 8=1$

D-dimer

$<1 \mu \mathrm{g} / \mathrm{mL}=0$

$>1 \mu \mathrm{g} / \mathrm{mL}=1$

Legend: BMI: body-mass index; F: female; M: male; mo: months. 